\title{
Multimodal MALDI Imaging Mass Spectrometry Reveals Spatially Correlated Lipid and Protein Changes in Mouse Heart with Acute Myocardial Infarction
}

\author{
Ibrahim Kaya ${ }^{1,2}$, Sanna Sämfors ${ }^{2,3 \dagger}$, Malin Levin ${ }^{3}$, Jan Borén*3, John S. Fletcher*2 \\ ${ }^{1}$ Department of Psychiatry and Neurochemistry, Sahlgrenska Academy at the University of Gothenburg, \\ Mölndal, Sweden \\ ${ }^{2}$ Department of Chemistry and Molecular Biology, University of Gothenburg, Sweden \\ ${ }^{3}$ Institute of Medicine, Department of Molecular and Clinical Medicine at University of Gothenburg and \\ Sahlgrenska University Hospital, Gothenburg, Sweden
}

\begin{abstract}
Acute myocardial infarction (MI) is a cardiovascular disease which remains a major cause of morbidity and mortality worldwide despite advances in its prevention and treatment. During acute myocardial ischemia, the lack of oxygen switches the cell metabolism to anaerobic respiration, with lactate accumulation, ATP depletion, $\mathrm{Na}^{+}$and $\mathrm{Ca}^{2+}$ overload and inhibition of myocardial contractile function, which drastically modifies the lipid, protein and small metabolite profile in the myocardium. Imaging mass spectrometry (IMS) is a powerful technique to comprehensively elucidate the spatial distribution patterns of lipids, peptides, and proteins in biological tissue sections. In this work, we demonstrate an application of multimodal chemical imaging using matrix-assisted laser desorption/ionization mass spectrometry (MALDI-MS) which provided comprehensive molecular information in situ within the same mouse heart tissue sections with myocardial infarction. MALDI-IMS (at $30 \mu \mathrm{m}$ per pixel) revealed infarct-associated spatial alterations of several lipid species of sphingolipids, glycerophospholipids, lysophospholipids and cardiolipins along with the acyl carnitines. Further, we performed trimodal MALDI-IMS (IMS3) where dual polarity lipid imaging was combined with subsequent protein MALDI-IMS analysis (at $30 \mu \mathrm{m}$ per pixel) within the same tissue sections which revealed accumulations of core histone proteins $\mathrm{H} 4, \mathrm{H} 2 \mathrm{~A}$ and $\mathrm{H} 2 \mathrm{~B}$ along with post-translational modification products, acetylated $\mathrm{H} 4$ and $\mathrm{H} 2 \mathrm{~A}$ on the borders of the infarcted region. This methodology allowed us to interpret the lipid and protein molecular pathology of the very same infarcted region in a mouse model of myocardial infarction. Therefore, the presented data highlight the potential of multimodal MALDI imaging mass spectrometry of the same tissue sections as a powerful approach for simultaneous interrogation of spatial infarct-associated lipid and protein changes of myocardial infarction.
\end{abstract}

\section{SUPPORTING INFORMATION}

\section{*Corresponding Authors}

John S. Fletcher, Jan Borén

john.fletcher@.chem.gu.se

jan.boren@wlab.gu.se 


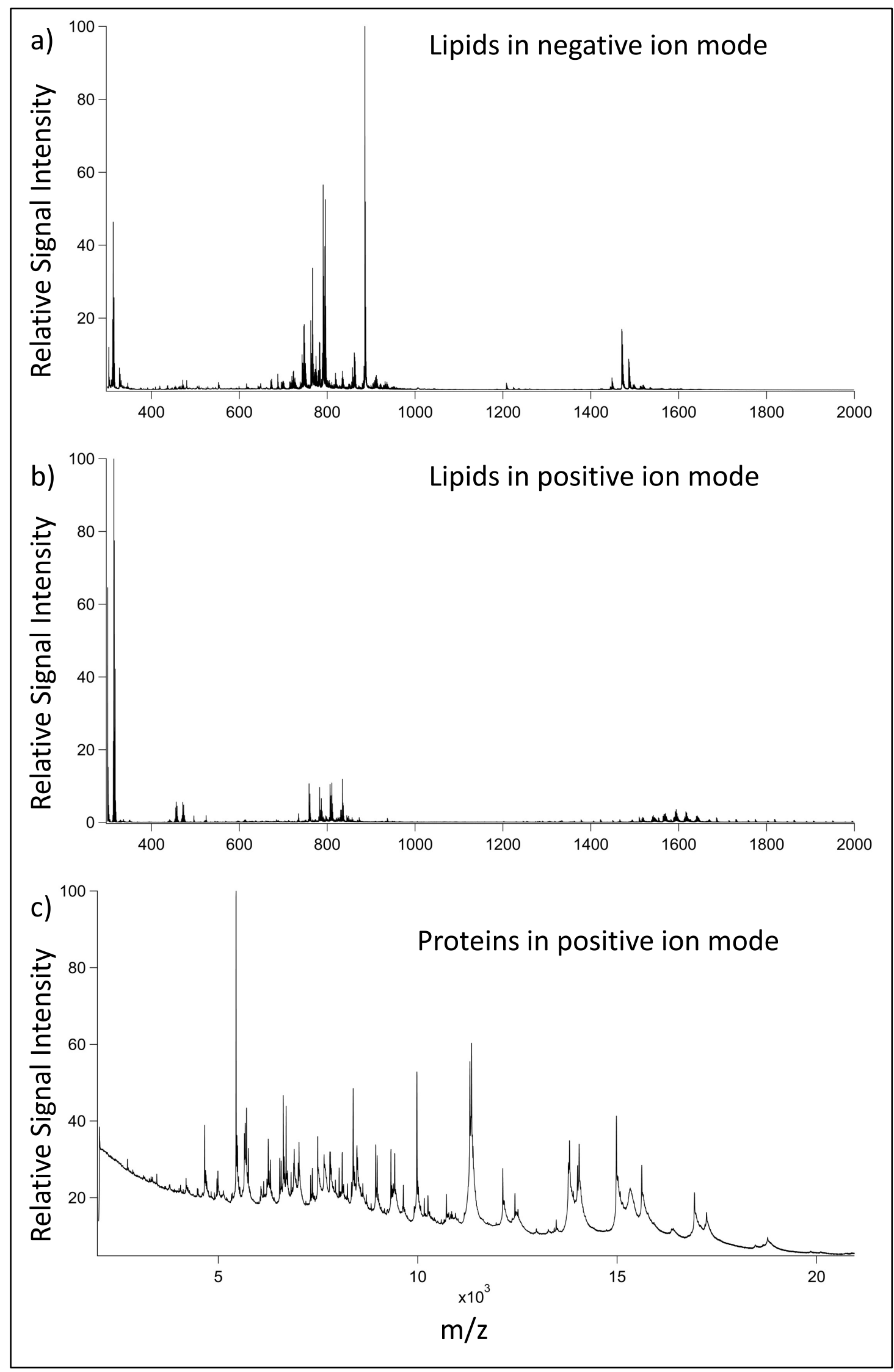

Supporting Information Figure S-1. Representative multimodal MALDI-MS spectra from a single coronal mouse heart tissue section with myocardial infarction. A) Reflective negative ion mode lipid spectrum, B) reflective positive ion mode lipid spectrum, and C) linear positive ion mode protein spectrum. 


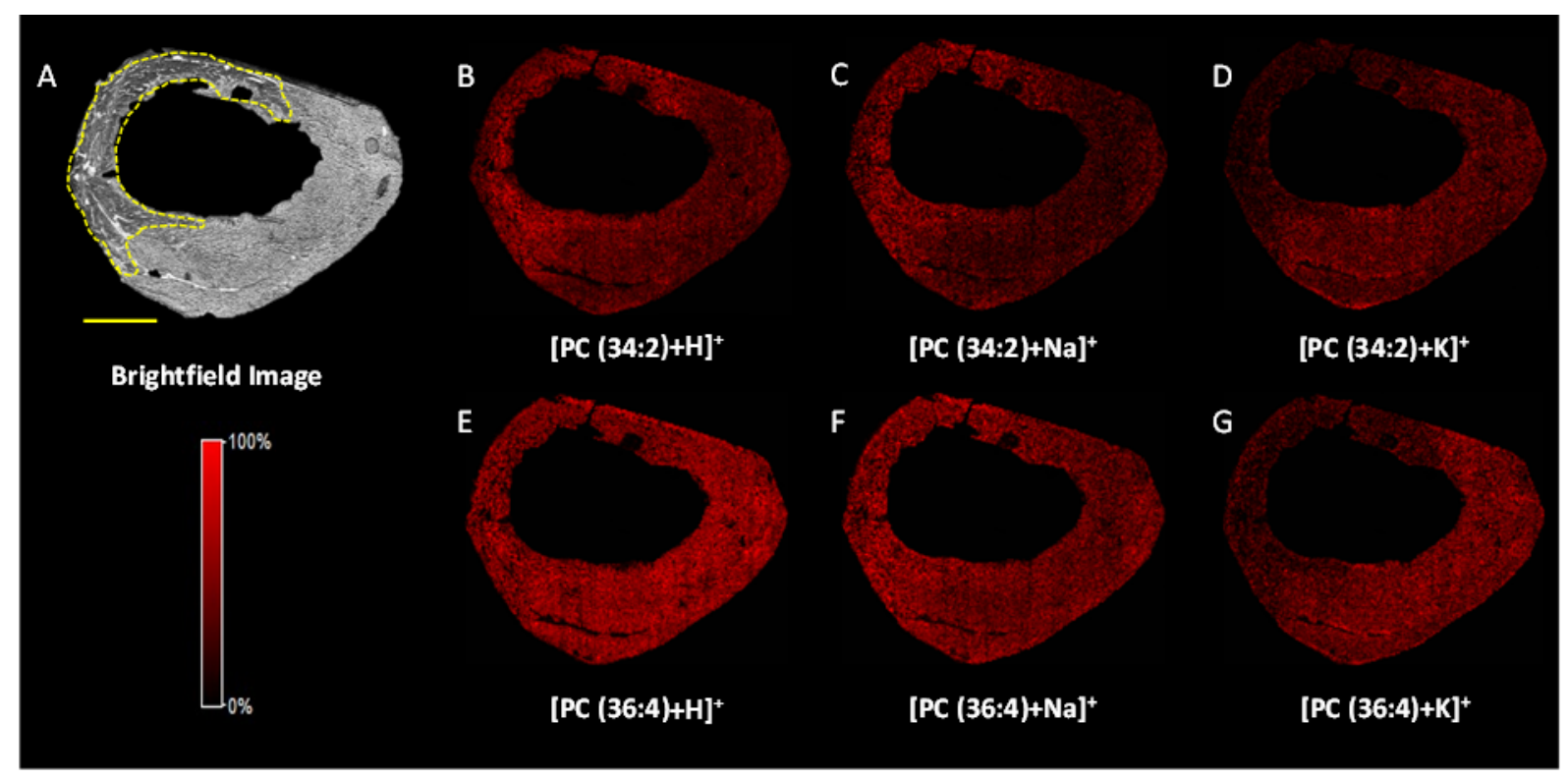

Supporting Information Figure S-2. Infarct-associated accumulation sodium adduct ion species and depletion of potassium adduct ion species of phosphatidylcholines (PCs) revealed by positive polarity MALDI mass spectrometry imaging (at $30 \mu \mathrm{m}$ per pixel spatial resolution) of coronal tissue section of mouse heart with myocardial infarction. A) Brightfield optical image of the analyzed tissue section to reveal the tissue morphology and infarcted region in yellow dashed line area. Ion images (in red) of PCs in positive ion mode reveals accumulations of B) PC (34:2)+H (m/z 758.5), C) PC (34:2)+Na (m/z 780.5), E) PC (36:4)+H (m/z 782.5), F) $\mathrm{PC}(36: 4)+\mathrm{Na}(\mathrm{m} / \mathrm{z} 804.5)$ and depletions of D) $\mathrm{PC}(34: 2)+\mathrm{K}(\mathrm{m} / \mathrm{z}$ 796.4) and $\mathrm{G}) \mathrm{PC}(36: 4)+\mathrm{K}(\mathrm{m} / \mathrm{z} 820.6)$. Scale bar in panel $\mathrm{A}$ is $1000 \mu \mathrm{m}$.

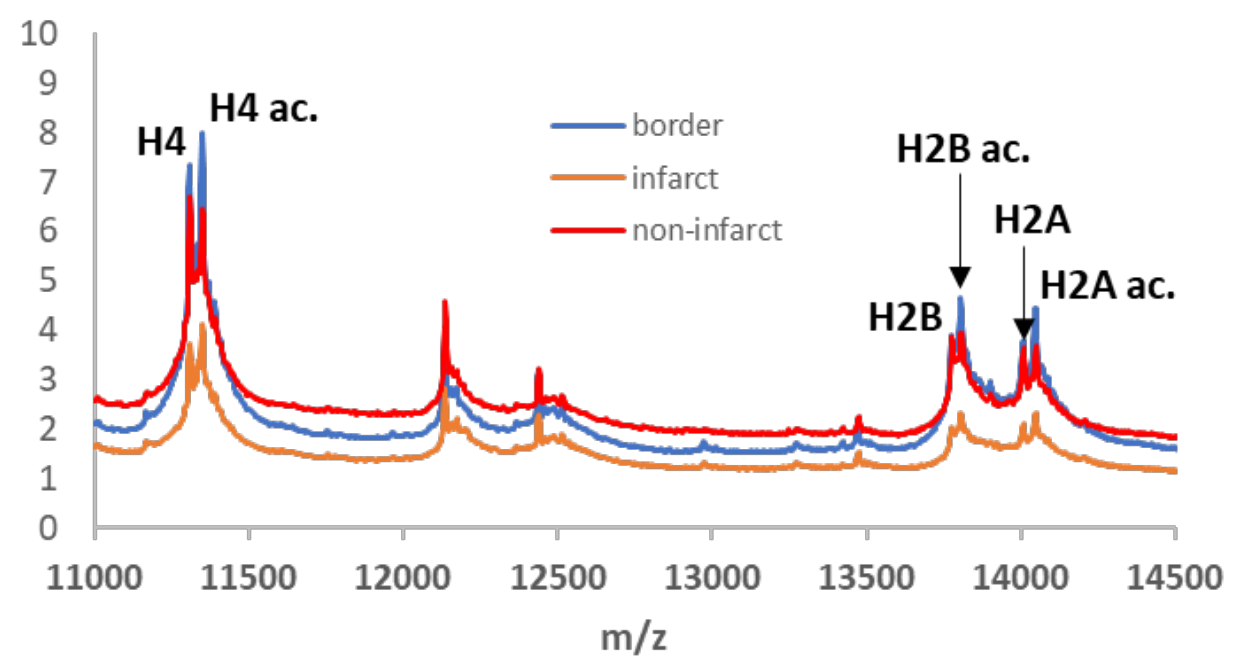

Supporting Information Figure S-3. Protein spectra of MALDI analysis of $24 \mathrm{~h}$ post infarction infarcted heart showing the difference in spectra when different regions of interest have been selected (border, infarct and non-infarcted region). The border and infarct region show the same ratio between the unmodified histone and the acetylated form, while the ratio is different for the non-infarcted region. 